\title{
A Localized Enantioselective Catalytic Site on Short DNA Sequences and their Amphiphiles
}

\author{
Jun Guo, ${ }^{[a], ~[+] ~ D a n y u ~ W a n g, ~}{ }^{[a], ~[b], ~[+] ~ E v a n g e l i a ~ P a n t a t o s a k i, ~}{ }^{[c]}$ Huihui Kuang, ${ }^{[a]}$ George K. \\ Papadopoulos, [c], [d] Michael Tsapatsis*[a], [b], [e] and Efrosini Kokkoli*[a], [b] \\ [a] Dr. J. Guo, D. Wang, Dr. H. Kuang, Prof. Dr. M. Tsapatsis, Prof. Dr. E. Kokkoli \\ Institute for NanoBioTechnology, Johns Hopkins University, Baltimore, MD 21218 (USA) \\ E-mail: tsapatsis@jhu.edu \\ E-mail: kokkoli@jhu.edu \\ [b] D. Wang, Prof. Dr. M. Tsapatsis, Prof. Dr. E. Kokkoli \\ Department of Chemical and Biomolecular Engineering, Johns Hopkins University, Baltimore, MD 21218 (USA) \\ [c] Dr. E. Pantatosaki, Prof. Dr. G. K. Papadopoulos \\ School of Chemical Engineering, National Technical University of Athens, 15780 Athens (Greece) \\ [d] Prof. Dr. G. K. Papadopoulos \\ Institute for Medical Engineering and Science, Massachusetts Institute of Technology, Cambridge, MA 02139 (USA) \\ [e] Prof. Dr. M. Tsapatsis \\ Applied Physics Laboratory, Johns Hopkins University, Laurel, MD 20723 (USA) \\ [+] \\ These authors contributed equally to this work.
}

\begin{abstract}
A DNA-based artificial metalloenzyme (ArM) consisting of copper (II) complex of 4,4'-dimethyl-2,2'-bipyridine (dmbipy-Cu) bound to double-stranded DNA (dsDNA) as short as 8 base pairs with only two contiguous central $G \cdot C$ pairs ( $G$ for guanine and $C$ for cytosine), catalyzes highly enantioselective Diels-Alder reaction in water. Molecular simulations indicate that these minimal sequences provide a single site where dmbipy-Cu is groove-bound and able to function as an enantioselective catalyst. Enantioselective preference inverses when D-DNA is replaced with LDNA. When the DNA is conjugated to a hydrophobic tail, the obtained ArMs exhibit enantioselective performance in a methanol-water mixture superior to that of non-amphiphilic dsDNA, and dsDNA-amphiphiles with more complex $\mathrm{G} \cdot \mathrm{C}$-rich sequences.
\end{abstract}

Keywords: artificial metalloenzyme $\bullet$ DNA catalysis $\bullet$ asymmetric catalysis $\bullet$ catalysis in methanol-water $\bullet$ DNAamphiphile

\section{Introduction}

Artificial metalloenzymes (ArMs) can combine the chiral environment of biomolecular scaffolds with the catalytic properties of a transition metal. ${ }^{1-6}$ Since the introduction of DNA-based ArMs in $2005,{ }^{7}$ enantioselective catalysis by metal ions or metal ion complexes assembled on a DNA scaffold has been demonstrated for Diels-Alder, ${ }^{7-13}$ FriedelCrafts alkylation, ${ }^{14-18}$ Michael addition, ${ }^{19-22}$ and other reactions ${ }^{23-30}$ Despite progress in correlating the catalytic performance of DNA-ArMs with their DNA sequence, ${ }^{8-12,15-18,21,24-28,30}$ pinpointing which of the many possible chiral microenvironments created by the interaction between the DNA and the bound metal, or metal complex, is responsible for enantioselective catalysis, remains elusive. As a result, our ability to design catalysts based on minimal DNA sequences with localized catalytic sites is limited. Minimal DNA sequences may enable cost-effective implementation of DNA scaffolds as well as their facile incorporation in extended constructs, like DNA-amphiphiles, aiming at efficient catalysis in complex solvent environments. Here, it is demonstrated that DNA-ArMs consisting of a $\mathrm{Cu}(\mathrm{II})$ complex with 4,4'-dimethyl-2,2'-bipyridine (dmbipy-Cu) bound to minimal dsDNA sequences, containing only two contiguous $\mathrm{G} \cdot \mathrm{C}$ base pairs, can be as highly active and selective as ArMs based on more extended DNA sequences. Based on these findings and molecular simulations of dmbipy-Cu interactions with DNA, a single catalytic site is proposed. Moreover, to establish possible practical implications, it is shown that the minimal TTTGGTTT and AAACCAAA DNA sequences can be conjugated to hydrophobic tails to create DNA-amphiphile ArMs, which in methanol-water solvents outperform their corresponding DNA ArMs, as well as DNA-amphiphile ArMs based on the G-rich sequence HT21. 


\section{Results and Discussion}

The Diels-Alder reaction between aza-chalcone (1a) and cyclopentadiene (2) was used to probe the catalytic performance of different dsDNA sequences (Figure 1 and Figure S1). There are four possible products for (3phenylbicyclo[2.2.1]hept-5-en-2-yl)(pyridin-2-yl)methanone (3a): Re-endo, Si-endo, Re-exo and Si-exo (Scheme S1) out of which the two endo enantiomers are dominant. ${ }^{31,32}$ The ability for enantioselectivity by the DNA-ArM catalyst is determined by the enantiomeric excess (ee\%) of the Si-endo versus Re-endo. ${ }^{3}$ 4,4'-dimethyl-2,2'bipyridine (dmbipy) was selected as the achiral ligand to bridge the $\mathrm{Cu}(\mathrm{II})$ catalytic center and the dsDNA scaffold. ${ }^{33}$ We include for comparison the performance of the natural salmon testes DNA (st-DNA) and a G•C-rich dsDNA sequence composed of the 21 nucleotide G-rich human telomeric sequence HT21. The ArM catalysts based on stDNA and HT21 exhibit high conversion and enantioselectivity with preference for the Si-endo (Figure 1) in agreement with expectations from a previous report. ${ }^{8}$

dsDNA G•C-rich sequences have been verified as active and enantioselective catalysts for the Diels-Alder reaction of (1a) and (2). ${ }^{8}$ However, the presence of multiple secondary structures in samples of HT21 and its complementary sequence, like anti-parallel G-quadruplex, i-motif and B-form, as well as variations among independently produced samples (i.e., prepared on different days using identical procedure) are evident in circular dichroism (CD) spectra (Figure S2), ${ }^{34}$ and make efforts to identify catalytic sites a complicated task. Our results are in agreement with reports from the literature where they showed that a completely hydrogen bonded one-to-one complex from a double-stranded polyG-polyC sample had to be prepared enzymatically, as otherwise the sequences could also form self-complexes. ${ }^{35}$ Therefore, in order to narrow down the catalytically active chiral microenvironments in the DNA duplex and explain the origin of asymmetric catalysis achieved by the $\mathrm{G} \cdot \mathrm{C}$-rich dsDNA-based ArMs, we tested a series of synthetic 21-base pair dsDNA sequences including the "21" sequence, which consists of 21 base pairs of adenine $(A)$ and thymine $(T)$, the "21-6", "21-6, 7", "21-6, 7, 8" and "21-6, 7, 8, 9"

Diels-Alder Reaction
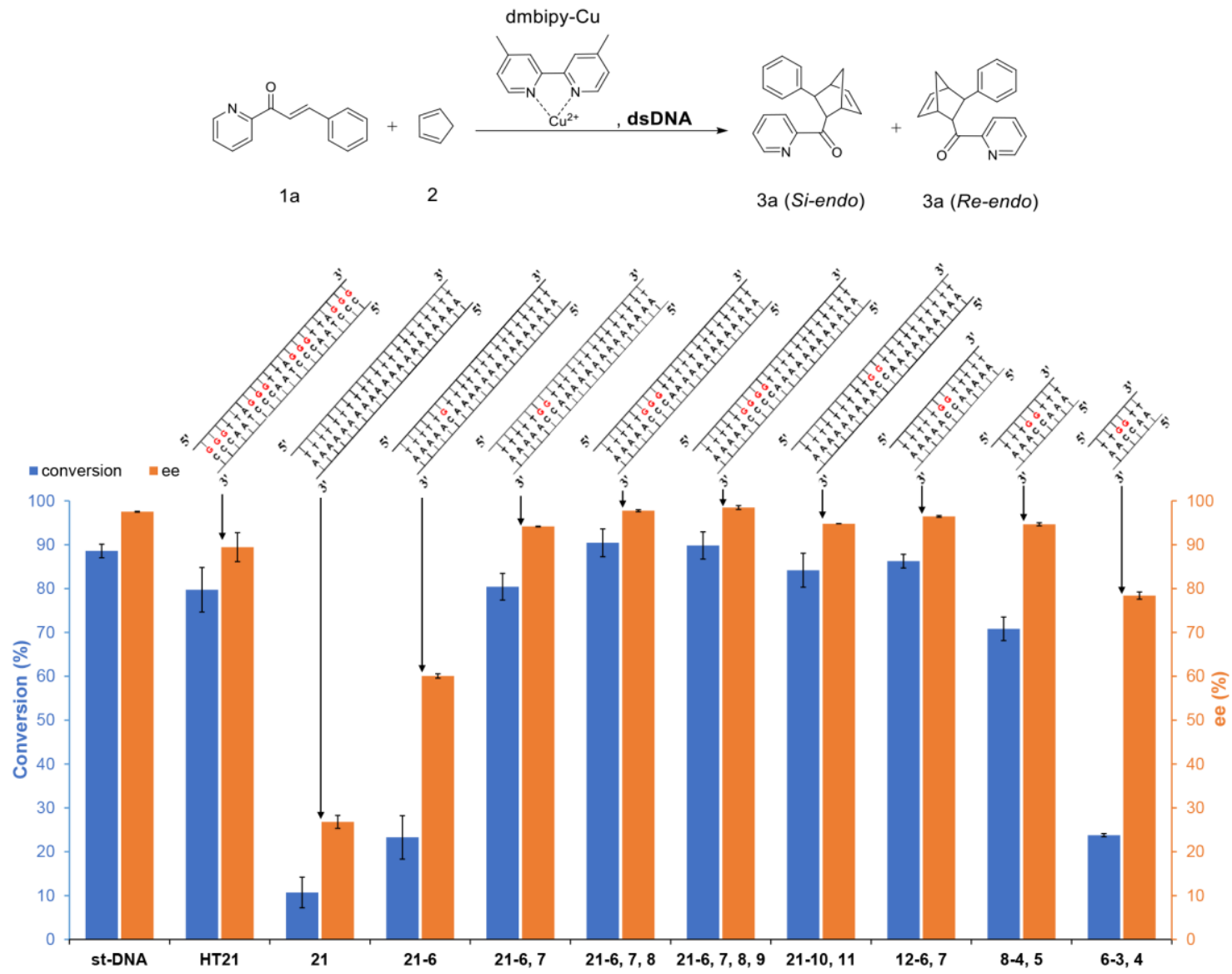

Figure 1. Conversion and ee\% for Diels-Alder reaction catalyzed by different dsDNA sequences with dmbipy-Cu. ee\% $=(\mathrm{moles}$ of $\mathrm{Si}$-endo - moles of $R e$-endo) / (moles of Si-endo + moles of Re-endo) $\times 100 \%$. All reactions were carried out in MOPS (20mM, pH 6.5) at $4{ }^{\circ} \mathrm{C}$ for $3 \mathrm{~h}$, st-DNA: $0.68 \mathrm{mg} / \mathrm{mL}(1.05 \mathrm{mM} \mathrm{base} \mathrm{pairs);}$ synthetic dsDNA: $50 \mu \mathrm{M}$; [dmbipy-Cu]: $50 \mu \mathrm{M}$; aza chalcone (1a): $1 \mathrm{mM}$; cyclopentadiene: $5.6 \mu \mathrm{L}$ (67 eq.). See experimental section (SI) for reaction procedure details. All data are averaged over three independent experiments. Parameters were determined for all displayed products by high performance liquid chromatography (HPLC) analysis on a chiral stationary phase. The conversion and ee were not detectable in the absence of dsDNA under the above cited conditions (longer times were required to achieve detectable conversion). 
sequences, which contain one to four contiguous $G \cdot C$ pairs (see Table $S 1$ and Scheme $S 2$ for naming the DNA sequences used in this study). The CD results confirmed that all DNA sequences used in Figure 1 and $S 1$ hybridized in the buffer used for catalysis and that the presence of dmbipy-Cu had no notable effect on the secondary structure of the dsDNA (Figure S4-S16). For comparison, the CD of st-DNA is shown in Figure S3. Sequence "21" showed four peaks between 245-285 nm (Figure S4), two maxima at 260 and $282 \mathrm{~nm}$ and two minima at 248 and $267 \mathrm{~nm}$ ), characteristic of B' heteronomous DNA-form adopted by poly(A)-poly(T) sequences. ${ }^{34,36}$ The presence of just one $\mathrm{G}$ has been shown to interrupt the A-track by decreasing the ellipticity at $260 \mathrm{~nm}$, as shown in Figure S5. ${ }^{36,37}$

It has been stated earlier that three contiguous $\mathrm{G}$ are correlated with high ee\% for the Diels-Alder reaction of (1a) and (2). ${ }^{8}$ Indeed, while the "21" sequence (consisting only of A•T pairs) was almost inert towards this reaction, after introducing one $\mathrm{G} \cdot \mathrm{C}$ pair to the duplex, ee increased from $27 \%$ to $60 \%$. When two contiguous $\mathrm{G} \cdot \mathrm{C}$ pairs are present in the " 21 " sequence, a notable increase in both conversion and enantioselectivity was observed for the "21-6, 7" sequence (94\% ee with preference for Si-endo at $80 \%$ aza-chalcone conversion). With the dsDNA sequences containing three or four $\mathrm{G} \cdot \mathrm{C}$ base pairs in a row, highly active hybrid catalysts were acquired, which provided Si-endo isomer with excellent enantioselectivities (98\% for both "21-6, 7, 8" and "21-6, 7, 8, 9"). These results indicate that in order to obtain a highly enantioselective ArM catalyst for the Diels-Alder reaction of (1a) and (2), a minimum of two contiguous $G \cdot C$ pairs is necessary and sufficient.

The position of the two G.C pairs is consequential. A comparison among 21-base pair dsDNA sequences containing two $\mathrm{G} \cdot \mathrm{C}$ base pairs at different positions is shown in Figure S1. A "21-6, 16" sequence with two separate $G \cdot C$ pairs provided only moderate enantioselectivity $(68 \%)$ further emphasizing the importance for the $G \cdot C$ pairs to be contiguous. When the two contiguous $\mathrm{G} \cdot \mathrm{C}$ pairs were moved to the end of the sequence ("21-20, 21"), the enantioselectivity dropped to $21 \%$. However, after moving the two G.C pairs by two nucleotides inwards ("21-18, $19 ")$, the ee $\%$ was restored to $90 \%$. By moving the two contiguous G.C pairs further toward the center zone, both conversion and enantioselectivity were increased again, with the best results given by the "21-10, 11" sequence ( $84 \%$ conversion and $95 \%$ ee). The significant effect of the two $G \cdot C$ pair's position on the catalytic results indicates sensitivity of the enantioselectivity to the chiral microenvironment created by the binding position of the bi-pyridine complex to the dsDNA scaffold.

Molecular dynamics (MD) simulations for the binding of dmbipy-Cu on two representative sequences: "21" and "21-10, 11" in ionic aqueous solution were conducted (details in SI). ${ }^{38}$ The MD simulations show that the dmbipyCu complex (Figure S17) in both sequences binds to the minor groove of DNA without intercalating its double helix. As shown from the time evolution of the distance between the dmbipy-Cu and the DNA in Figure S18, the molecules remain bound along the entire equilibrated MD trajectory. Figure S19 presents the rise per base pair along the helix axis, namely the distance between adjacent bases in the same DNA strand, computed over the entire MD trajectories. It is seen that, in both sequences, the average rise per base pair fluctuates around the crystallographic value denoting no increase, and thus no dmbipy-Cu intercalation. In Figure S20, the probability of finding dmbipy$\mathrm{Cu}$ at various distances from the minor or major groove base atoms indicates that the dmbipy-Cu is a minor groove binder in both sequences. In particular, in "21", the dmbipy-Cu complex resides in the minor groove covering five nucleotide pairs, from T8 to T12, while in "21-10, 11" it bounds in the vicinity of the G•C pairs covering a fournucleotide sequence, from T7 to G10, as shown in Figure S21 depicting the minimum distance between the dmbipy$\mathrm{Cu}$ and each of the 21 DNA nucleotide pairs.

The dmbipy-Cu to DNA binding pattern was further investigated by computing the probability of finding the copper atom or the pyridine methyl carbons at various distances from the minor groove base atoms, as depicted in Figure 2a and 2b, and Figure S22 (details in SI). In "21", the position of the dmbipy-Cu was found to be highly localized, with both methyl carbons residing at short distances from the minor groove base atoms (cumulative probability $\mathrm{p}(0.3 \leq \mathrm{s} \leq 0.5)$ approximately 0.93 and 0.80 for $\mathrm{C} 15$ and $\mathrm{C} 9$ respectively). Also, copper lies at large distances into the solvent $(p(0.7 \leq s \leq 1.1)$ about 0.90$)$. These findings indicate that the ligand orients both pyridine methyls into the groove as shown in the indicative configuration rendered from the MD trajectory (Figure 2c). This configuration is enabled by the quasi-planar structure of dmbipy-Cu which allows for the fit of the molecule in between the minor groove walls.

Contrarily, in "21-10, 11", the respective distributions are considerably wider denoting enhanced dmbipy-Cu mobility, in agreement with previous suggestions regarding dmbipy-Cu and G.C-rich st-DNA. ${ }^{39}$ We found that the pyridine methyls may be located into, or out ( $\mathrm{p}(0.5 \leq \mathrm{s} \leq 1.2)$ approximately 0.70 and 0.80 for $\mathrm{C} 15$ and $\mathrm{C}$, respectively), of the minor groove. Also, the copper atom may reside into the groove or into the DNA hydration shell close to the edge of the groove ( $p(0.5 \leq s \leq 0.7)$ value about 0.55$)$. The above findings show that the dmbipy-Cu may direct one of its pyridine rings in the groove and the other in the solvent with the copper atom close to the minor groove edge (Figure 2d). 

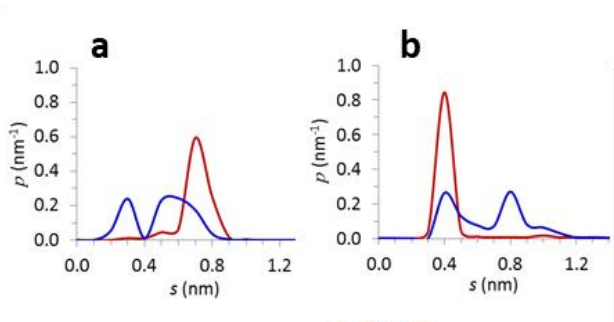

c

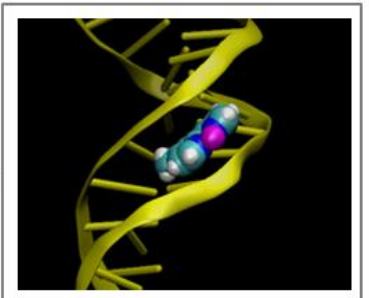

d

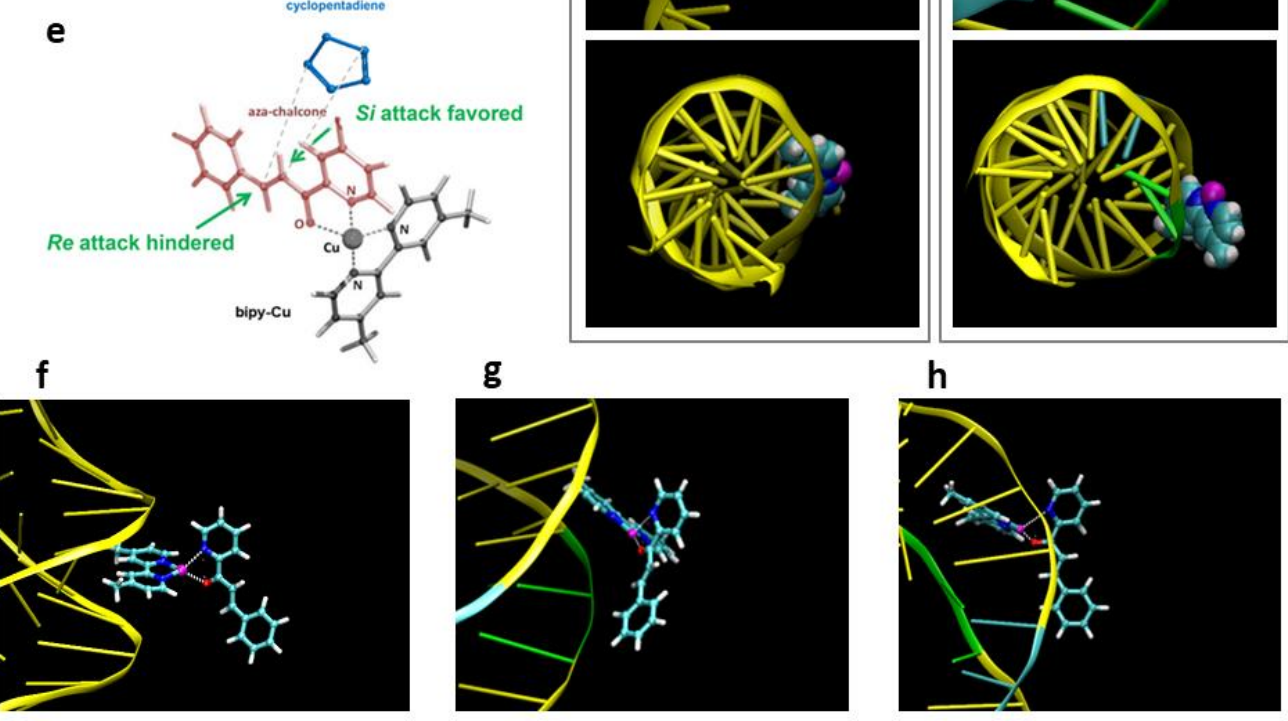

Figure 2. Probability density functions of finding the copper atom (a), and pyridine methyl carbon $\mathrm{C} 15$ (b), of the dmbipy-Cu, at various distances from the minor groove base atoms in " 21 " (red) and " $21-10,11$ " (blue) sequences, computed over the equilibrated MD trajectory (for a similar graph for pyridine methyl carbon C9 see Figure S22; the notation of carbon atoms is indicated in Figure S17). Indicative configurations rendered from the MD trajectory depicting the dmbipy-Cu binding to the DNA in "21" (c) and "21-10, 11" (d). The reactant (1a), added by following the bidentate coordination pattern of the enone to the copper atom of the dmbipy$\mathrm{Cu}$ as shown in (e), is depicted in (f) for "21", and ( $\mathrm{g}, \mathrm{h})$ for "21-10, 11 ". ( $\mathrm{g}, \mathrm{h}$ ) depict the same configuration in two rotated views for clarity. Water molecules in the rendered pictures are omitted for clarity; the same configurations including the water molecules are depicted in Figure S24. Color code for the dmbipy-Cu and reactant (1a): Cu (magenta), $\mathrm{N}$ (blue), $\mathrm{C}$ (cyan), $\mathrm{H}$ (white), $\mathrm{O}$ (red); color code for the DNA: guanine (green), cytosine (cyan), adenine and thymine (yellow).

The distinct configuration for dmbipy-Cu-dsDNA "21-10, 11" is due to the presence of the G・C pairs, and the $\mathrm{G} \cdot \mathrm{C}$-induced local widening of the minor groove; while on the contrary, the narrow minor groove of the pure $\mathrm{A} \cdot \mathrm{T}$ sequence "21" does not enable such behavior (Figure S23). In other words, the groove size is sequence-dependent, and the widening is not induced by the dmbipy-Cu, as shown by calculations of the minor groove width for the two initial DNA structures (Figure S23). The above computations are in agreement with experiments showing that A•Trich sequences exhibit narrower minor grooves than G•C-rich DNA.40, 41

The dissimilar binding patterns of the dmbipy-Cu in "21" and "21-10, 11" result in a different hydration level for copper, as revealed by the computed radial distribution functions shown in Figure S25a. In "21-10, 11", the coordination of water molecules around copper is partially restricted because of the "volume" occupied by the DNA, which reduces the accessible surface of the copper atom by the solvent; in "21-10, 11" the Cu atom was found to coordinate with the oxygen atoms of the DNA phosphate groups with higher probability than in the "21" sequence (cumulative probability $\mathrm{p}(\mathrm{s} \leq 0.3)$ approximately 0.7 and 0.4 respectively) (see Figure $\mathrm{S} 25 \mathrm{~b}$ ). Changes in the coordination of $\mathrm{Cu}$ in the presence of DNA have been suggested earlier. ${ }^{42}$

As shown in Figure 2e, the experimentally determined catalytic outcome requires a preferential acceleration of the cyclopentadiene attack through the Si-face of (1a) when attached to dmbipy-Cu bound to "21-10, 11" while no such acceleration is observed for " 21 ". We propose that this is accomplished by the distinct sequence-dependent binding patterns of the dmbipy-Cu in the sequences " 21 " and "21-10, 11" as predicted by MD, which in turn can impose different orientations of the reactant (1a) with respect to DNA upon its bidentate coordination to the copper atom. Here, we do not undertake the challenging task of simulating the coordination of (1a) with dmbipy-Cu and dsDNA. Instead, we consider the dmbipy-Cu and dsDNA configurations determined by MD and assume that the bidentate coordination of (1a) occurs with the two quasi-planar molecules orienting their planes in a perpendicular fashion. ${ }^{43}$ We show schematically in Figure $2 f$ and Figures $2 g$, $2 \mathrm{~h}$, possible configurations of DNA-dmbupy-Cu-(1a) based on the above assumption (see also Figure S26 for alternative configurations). In the "21" sequence, both faces of the (1a) are away from the DNA double helix and in contact with the bulk solvent (Figure 2f), whereas in the sequence "21-10,11", one face of the (1a) is close to the DNA double helix with the other face being in contact with the bulk solvent (Figures $2 \mathrm{~g}, 2 \mathrm{~h}$ ). Consistently with the experimental findings, the "21" configuration cannot 
provide preference for Si-face vs. Re-face attack of (2) to (1a). In the "21-10, 11" configuration shown in Figures $2 \mathrm{~g}$, $2 \mathrm{~h}$, the Si-face is near the DNA. Therefore, in this configuration, the Si-face attack of (2) to (1a) will take place with (2) present in the pocket created between (1) and the DNA. In a recent study of the same reaction catalyzed by $\mathrm{Cu}^{2+}$-adenosine triphosphate, density functional theory gas phase calculations suggested that Si-face attack was favored due to hydrogen bonding of (2) with phosphate oxygen atoms leading to a reduced energy barrier for the cycloaddition. ${ }^{44}$ One possible explanation for our system is that DNA-(2) interactions in the pocket created between the Si-face of 1(a) and dsDNA lead to a free energy transition state reduction causing the observed rate increase and high ee observed experimentally for "21-10, 11". An alternative explanation for the observed ee, is that the relevant configuration for catalysis is with (1a) oriented as shown in Figure S26, i.e., with (1a) rotated by $180^{\circ}$ compared to its configuration in Figures $2 \mathrm{~g}, 2 \mathrm{~h}$. In this configuration, the Re-face of (1a) is near the DNA and is being shielded against $R e$-face attack. In this scenario the rate acceleration observed experimentally can be attributed to an increase in local concentration as suggested in a previous study. ${ }^{42}$ At this point, without further simulations, we cannot provide definite support for one of the two aforementioned scenarios as they can both be consistent with the observed ee in favor of the Si-endo isomer.

The optimized "21-10, 11" sequence was chosen and truncated to get analogous sequences with 12, 8 , and 6 base pairs (Figure 1). The catalytic results indicate that, to maintain high enantioselectivity, the dsDNA sequence with two central contiguous $\mathrm{G} \cdot \mathrm{C}$ base pairs can be as short as 8 base pairs (" $8-4,5$ "). The melting temperature (Figure S27) of "8-4,5" was determined to be $25.6 \pm 0.6{ }^{\circ} \mathrm{C}$ while that of " 8 ", HT21 and st-DNA are $12.7 \pm 0.6{ }^{\circ} \mathrm{C}$, $65.7 \pm 0.6{ }^{\circ} \mathrm{C}$ and $85.0 \pm 0.1^{\circ} \mathrm{C}$ indicating that they are all stable at the reaction temperature of $4{ }^{\circ} \mathrm{C}$. However, when the sequence was truncated to 6 base pairs ("6-3, 4"), lower conversion (24\%) and ee (78\%) were observed. Although the role of DNA sequence ${ }^{8,45,46}$ and length ${ }^{16}$ has been discussed before, to our knowledge, the highly enantioselective performance of only two contiguous $G \cdot C$ base pairs in a sequence as short as eight nucleotides has not been demonstrated. Our short dsDNA sequences are comparable in size and performance with catalysts consisting of dmbipy-Cu and short (14-nucleotide) sequences that fold into hairpin structures containing a minimum of three G.C pairs. ${ }^{47}$ The simplicity of our system allowed us to propose the active site configuration by performing statistical mechanics modeling.

The binding constant of dmbipy-Cu is shown in Figure S28. The binding constant for st-DNA $\left((9.25 \pm 0.75)^{\star} 10^{3}\right.$ $\left.\mathrm{M}^{-1}\right)$ is consistent with that reported by the Roelfes group $\left(7.26^{*} 10^{3} \mathrm{M}^{-1}\right) \cdot{ }^{8}$ According to our results, the dmbipy-Cu can bind strongly to both " 8 " ((9.99 \pm 0.79$\left.)^{*} 10^{3} \mathrm{M}^{-1}\right)$ and "8-4,5" $\left((10.34 \pm 1.03)^{*} 10^{3} \mathrm{M}^{-1}\right)$ sequences, indicating that the enantioselective catalytic performance differences (i.e., rate acceleration in favor of the Si-endo isomer) are not due to differences in binding but due to differences in configuration. This is consistent with the MD simulations discussed above.

The ability for inverting the enantioselectivity by switching from right-handed D-DNA to the left-handed L-DNA, introduced earlier, ${ }^{48,49}$ was confirmed for the "8-4, 5" sequence (HPLC chromatograms are shown in Figure S29). The left-handed "8-4,5" sequence was confirmed by contrasting the CD spectra of L-DNA (Figure S30) with the CD spectra of the natural right-handed D-DNA (Figure S11). As shown in Table 1 (entry 1 and entry 2), by switching the duplex backbone of "8-4, 5" from right-handed to left-handed and providing a microenvironment of opposite chirality, the enantioselectivity was reversed. When using D-DNA, cyclopentadiene addition from the Si-side is favored forming the Si-endo product, while for L-DNA, addition from the Re-side is favored to form the Re-endo product. By using equimolar amount of L-DNA and D-DNA, a racemic endo product was obtained (Table 1, entry 3 ) as expected based on identical catalytic activities of the mirror images of the catalytic sites.

Table 1. Conversion and ee\% for Diels-Alder reaction catalyzed by D-DNA and/or L-DNA with dmbipy-Cu. ${ }^{[a]}$

\begin{tabular}{cccccc}
\hline Entry & $\begin{array}{c}\text { D-DNA } \\
(\mu \mathrm{M})\end{array}$ & $\begin{array}{c}\text { L-DNA } \\
(\mu \mathrm{M})\end{array}$ & $\begin{array}{c}\text { Conversion } \\
(\%)\end{array}$ & endo/exo & ee (\%) $)^{[\mathrm{b}]}$ \\
\hline 1 & 50 & -- & 71 & $99 / 1$ & 95 \\
2 & -- & 50 & 63 & $99 / 1$ & -95 \\
3 & 25 & 25 & 45 & $98 / 2$ & -2 \\
\hline
\end{tabular}

[a] Same reaction conditions as in Figure 1. [b] ee\% $=($ moles of Si-endo - moles of Re-endo) $/($ moles of Si-endo + moles of Re-endo $) \times 100 \%$.

We also examined a series of 8-base pair variations (Figure S31), which further confirmed the importance of two contiguous $\mathrm{G} \cdot \mathrm{C}$ pairs. Hybridization and the presence of B-form conformation was verified for all of these dsDNA sequences by CD (Figure S11 and S32-S35). Activity and selectivity decreased by switching the $G$ and $C$ base groups of one of the two contiguous $G \cdot C$ pairs (group B, Figure S31). Further highlighting the importance of 
two contiguous $G \cdot C$ pairs, a sequence of 8 alternating $G \cdot C$ pairs (group C, Figure S31) also exhibited reduced activity and selectivity.

The minimal sequences with a localized enantioselective site presented above are sufficient to match the performance of ArMs with more complicated sequences allowing for better assessment of the location of the catalytic site. Moreover, they may enable cost effective utilization of DNA by complementing other approaches towards practical applications like the ones using bound DNA on solid matrices. ${ }^{50,51}$ We demonstrate next a case where the performance of a minimal DNA sequence with $2 \mathrm{G} \cdot \mathrm{C}$ pairs (" $8-4,5$ " sequence) exceeds the performance of the HT21 sequence with $4 \mathrm{G} \cdot \mathrm{C}$ triplets, highlighting the practical significance of the minimal sequences introduced herein. The "8-4, 5" sequence and the HT21 sequence were used in a methanol-water mixture (v/v=50/50) for the Diels-Alder reaction of (1a) and (2). As shown in Figure 3, both dsDNA sequences lost their enantioselective ability. This finding is in agreement with previous reports that the highly enantioselective DNA-based ArM has limited tolerance towards organic co-solvents. ${ }^{52-54}$

To recover the asymmetric catalytic performance of the synthetic dsDNA sequences in the methanol-water $(\mathrm{v} / \mathrm{v}=50 / 50)$ system, we introduce an additional driving force for assembly of the DNA strands. Earlier studies have demonstrated that conjugation of DNA to polyethylene glycol can improve its solubility, assembly and catalytic function in organic solvents, albeit without showing enantioselectivity. ${ }^{55}$ A hydrophobic tail was synthesized, ${ }^{56}$ and conjugated to both the 5' end of the ssDNA sequence and the 3' end of the complementary ssDNA sequence (Scheme S3). ${ }^{57}$ Successful conjugation was confirmed by matrix assisted laser desorption ionization-time of flight mass spectrometry (Table S2). CD spectra showed that tail conjugation did not have an effect on the secondary structure of the sequences in methanol-water (Figure S36-S37). "8-4, 5" and its amphiphile showed B-form conformation. HT21 and its amphiphile in methanol-water had a strong positive peak at $291 \mathrm{~nm}$ and a negative peak around $260 \mathrm{~nm}$, characteristic of anti-parallel G-quadruplex and i-motif. ${ }^{34}$ However, the presence of B-form cannot be excluded for HT21 as the negative hump around $245 \mathrm{~nm}$ could result from cancellation of a negative peak from B-form and a positive peak from anti-parallel G-quadruplex at this wavelength. The catalytic performance of the dsDNA amphiphiles in methanol-water $(\mathrm{v} / \mathrm{v}=50 / 50)$ system is shown in Figure 3 . We found that the enantioselectivity achieved by the "8-4, 5-amph." dsDNA amphiphiles (41\%) was significantly higher than that given by the "HT21-amph." (29\%). Further work is required to decipher the structure of the amphiphiles, the distribution of solvents and reactants and their effect on reaction rates and selectivities. However, this serves as a first example of an ArM based on a short dsDNA sequence with only two contiguous $G \cdot C$ pairs outperforming dsDNA with extra and more extended $\mathrm{G}$-tracts in a catalytic system that benefits from contiguous $\mathrm{G} \cdot \mathrm{C}$ pairs. The findings reported here, and in earlier studies, ${ }^{47}$ justify further exploration of minimal sequences with localized catalytic sites incorporated in ArMs, both from the fundamental and practical standpoint.

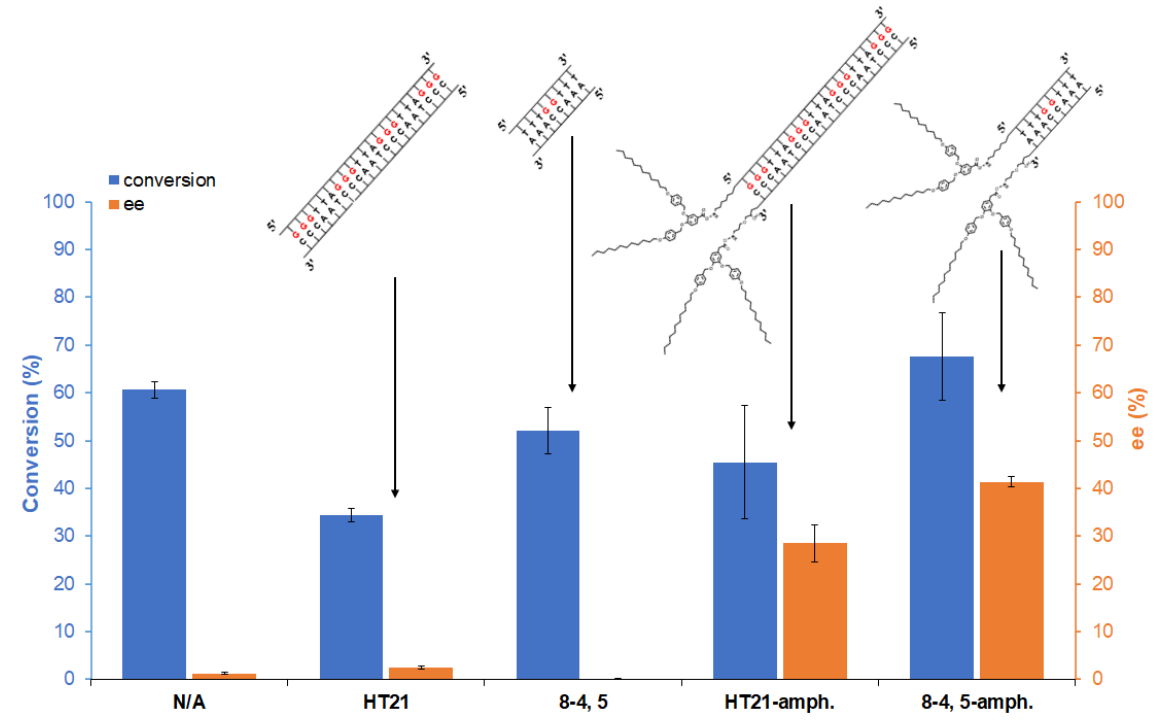

Figure 3. Conversion and ee\% of Diels-Alder reaction catalyzed by different dsDNA sequences with dmbipy-Cu in methanol-water (v/v = 50/50) mixture. All reactions were carried out in MOPS (20 mM, pH 6.5) at $4{ }^{\circ} \mathrm{C}$ for $48 \mathrm{~h}$, dsDNA: $15 \mu \mathrm{M}$; [dmbipy-Cu]: $15 \mu \mathrm{M}$; aza chalcone (1a): $1 \mathrm{mM}$; cyclopentadiene: $5.6 \mu \mathrm{L}$ (67 eq.). See experimental section (SI) for reaction procedure details. All data are averaged over three independent experiments. Parameters were determined for all displayed products by HPLC analysis on a chiral stationary phase. N/A means the reaction was performed in the absence of dsDNA. 


\section{Acknowledgements}

This work was partially funded by the Whiting School of Engineering and the Bloomberg Distinguished Professorship program of the Johns Hopkins University. E.P. acknowledges a grant of Scientific Excellence by the Hellenic Foundation for Research and Innovation (Project: B.1003-ENGETACT). G.K.P. acknowledges support by the European Union through a fellowship under H2020-MSC-IF (Project: 796794-ENGEMED), and by the Greek Research and Technology Network for CPU-time provided in the National HPC facility "ARIS". We would also like to thank the NMR core facility at the Department of Chemistry, the Center for Molecular Biophysics at Johns Hopkins University and Dr. Katherine Tripp for technical assistance with CD spectra.

\section{Conflict of interest}

The authors declare no conflict of interest.

\section{References}

1. Ringenberg, M. R.; Ward, T. R., Merging the best of two worlds: artificial metalloenzymes for enantioselective catalysis. Chem. Commun. 2011, 47, 8470-8476.

2. Wang, C.; Hao, M.; Qi, Q.; Dang, J.; Dong, X.; Lv, S.; Xiong, L.; Gao, H.; Jia, G.; Chen, Y., Highly Efficient Cyclic Dinucleotide Based Artificial Metalloribozymes for Enantioselective Friedel-Crafts Reactions in Water. Angew. Chem. Int. Ed. 2020, 59, $3444-3449$.

3. Wang, C.; Qi, Q.; Li, W.; Dang, J.; Hao, M.; Lv, S.; Dong, X.; Gu, Y.; Wu, P.; Zhang, W., A Cu (II)-ATP complex efficiently catalyses enantioselective Diels-Alder reactions. Nat. Commun. 2020, 11, 1-8.

4. Zhou, Z.; Roelfes, G., Synergistic catalysis in an artificial enzyme by simultaneous action of two abiological catalytic sites. Nat. Catal. 2020, 3, 289-294.

5. Lu, Y.; Yeung, N.; Sieracki, N.; Marshall, N. M., Design of functional metalloproteins. Nature 2009, 460, 855.

6. Hyster, T. K.; Ward, T. R., Genetic optimization of metalloenzymes: enhancing enzymes for non-natural reactions. Angew. Chem. Int. Ed. 2016, 55, 7344-7357.

7. Roelfes, G.; Feringa, B. L., DNA-based asymmetric catalysis. Angew. Chem. Int. Ed. 2005, 44, 3230-3232.

8. Boersma, A. J.; Klijn, J. E.; Feringa, B. L.; Roelfes, G., DNA-based asymmetric catalysis: sequence-dependent rate acceleration and enantioselectivity. J. Am. Chem. Soc. 2008, 130, 11783-11790.

9. Rosati, F.; Boersma, A. J.; Klijn, J. E.; Meetsma, A.; Feringa, B. L.; Roelfes, G., A kinetic and structural investigation of DNA-based asymmetric catalysis using first-generation ligands. Chem. Eur. J. 2009, 15, 9596-9605.

10. Park, S.; Okamura, I.; Sakashita, S.; Yum, J. H.; Acharya, C.; Gao, L.; Sugiyama, H., Development of DNA metalloenzymes using a rational design approach and application in the asymmetric Diels-Alder reaction. ACS Catal. 2015, 5, 4708-4712.

11. Wang, C.; Jia, G.; Zhou, J.; Li, Y.; Liu, Y.; Lu, S.; Li, C., Enantioselective Diels-Alder reactions with G-quadruplex DNA-based catalysts. Angew. Chem. Int. Ed. 2012, 51, 9352-9355.

12. Wilking, M.; Hennecke, U., The influence of G-quadruplex structure on DNA-based asymmetric catalysis using the G-quadruplexbound cationic porphyrin TMPyP4. Cu. Org. Biomol. Chem. 2013, 11, 6940-6945.

13. Roe, S.; Ritson, D. J.; Garner, T.; Searle, M.; Moses, J. E., Tuneable DNA-based asymmetric catalysis using a G-quadruplex supramolecular assembly. Chem. Commun. 2010, 46, 4309-4311.

14. García-Fernández, A.; Megens, R. P.; Villarino, L.; Roelfes, G., DNA-Accelerated Copper Catalysis of Friedel-Crafts Conjugate Addition/Enantioselective Protonation Reactions in Water. J. Am. Chem. Soc. 2016, 138, 16308-16314.

15. Boersma, A. J.; Feringa, B. L.; Roelfes, G., Enantioselective Friedel-Crafts reactions in water using a DNA-based catalyst. Angew. Chem. Int. Ed. 2009, 121, 3396-3398.

16. Park, S.; Ikehata, K.; Watabe, R.; Hidaka, Y.; Rajendran, A.; Sugiyama, H., Deciphering DNA-based asymmetric catalysis through intramolecular Friedel-Crafts alkylations. Chem. Commun. 2012, 48, 10398-10400.

17. Park, S.; Zheng, L.; Kumakiri, S.; Sakashita, S.; Otomo, H.; Ikehata, K.; Sugiyama, H., Development of DNA-based hybrid catalysts through direct ligand incorporation: Toward understanding of DNA-based asymmetric catalysis. ACS Catal. 2014, 4, 4070-4073.

18. Wang, C.; Li, Y.; Jia, G.; Liu, Y.; Lu, S.; Li, C., Enantioselective Friedel-Crafts reactions in water catalyzed by a human telomeric G-quadruplex DNA metalloenzyme. Chem. Commun. 2012, 48, 6232-6234.

19. Dey, S.; Jäschke, A., Tuning the stereoselectivity of a DNA-catalyzed Michael addition through covalent modification. Angew. Chem. Int. Ed. 2015, 54, 11279-11282.

20. Coquière, D.; Feringa, B. L.; Roelfes, G., DNA-based catalytic enantioselective Michael reactions in water. Angew. Chem. Int. Ed. 2007, 46, 9308-9311.

21. Megens, R. P.; Roelfes, G., DNA-based catalytic enantioselective intermolecular oxa-Michael addition reactions. Chem. Commun. 2012, 48, 6366-6368.

22. Punt, P. M.; Langenberg, M. D.; Altan, O.; Clever, G. H., Modular Design of G-Quadruplex MetalloDNAzymes for Catalytic C-C Bond Formations with Switchable Enantioselectivity. J. Am. Chem. Soc. 2021, 143, 3555-3561.

23. Fournier, P.; Fiammengo, R.; Jäschke, A., Allylic amination by a DNA-diene-iridium (I) hybrid catalyst. Angew. Chem. Int. Ed. 2009, 48, 4426-4429.

24. Boersma, A. J.; Coquière, D.; Geerdink, D.; Rosati, F.; Feringa, B. L.; Roelfes, G., Catalytic enantioselective syn hydration of enones in water using a DNA-based catalyst. Nat. Chem. 2010, 2, 991-995.

25. Yum, J. H.; Park, S.; Hiraga, R.; Okamura, I.; Notsu, S.; Sugiyama, H., Modular DNA-based hybrid catalysts as a toolbox for enantioselective hydration of alpha,beta-unsaturated ketones. Org. Biomol. Chem. 2019, 17, 2548-2553.

26. Hao, J.; Miao, W.; Cheng, Y.; Lu, S.-M.; Jia, G.; Li, C., Enantioselective Olefin Cyclopropanation with G-quadruplex DNA-based Biocatalysts. ACS Catal. 2020.

27. Duchemin, N.; Heath-Apostolopoulos, I.; Smietana, M.; Arseniyadis, S., A decade of DNA-hybrid catalysis: from innovation to comprehension. Org. Biomol. Chem. 2017, 15, 7072-7087. 
28. Mansot, J.; Vasseur, J. J.; Arseniyadis, S.; Smietana, M., alpha, beta-Unsaturated 2-Acyl-Imidazoles in Asymmetric Biohybrid Catalysis. ChemCatChem 2019.

29. Park, S.; Sugiyama, H., DNA as a chiral scaffold for asymmetric synthesis. Molecules 2012, 17, 12792-12803.

30. Park, S.; Sugiyama, H., DNA-based hybrid catalysts for asymmetric organic synthesis. Angew. Chem. Int. Ed. 2010, 49, $3870-3878$.

31. Otto, S.; Bertoncin, F.; Engberts, J. B., Lewis acid catalysis of a Diels- Alder reaction in water. J. Am. Chem. Soc. 1996, 118, 7702-

7707.

32. Otto, S.; Engberts, J. B., A systematic study of ligand effects on a Lewis-acid-catalyzed Diels- Alder reaction in water. Water-enhanced enantioselectivity. J. Am. Chem. Soc. 1999, 121, 6798-6806.

33. Roelfes, G.; Boersma, A. J.; Feringa, B. L., Highly enantioselective DNA-based catalysis. Chem. Commun. 2006, 635-637.

34. Kypr, J.; Kejnovská, I.; Renčiuk, D.; Vorličková, M., Circular dichroism and conformational polymorphism of DNA. Nucleic Acids Res. 2009, 37, 1713-1725.

35. Gray, D. M.; Bollum, F., A circular dichroism study of poly dG, poly dC, and poly dG: dC. Biopolymers: Original Research on Biomolecules 1974, 13, 2087-2102.

36. Gudibande, S. R.; Jayasena, S. D.; Behe, M. J., CD studies of double-stranded polydeoxynucleotides composed of repeating units of contiguous homopurine residues. Biopolymers 1988, 27, 1905-1915.

37. Brahms, S.; Brahms, J. G., DNA with adenine tracts contains poly(dA).poly(dT) conformational features in solution. Nucleic Acids Res. 1990, 18, 1559-1564.

38. Pantatosaki, E.; Papadopoulos, G. K., Binding Dynamics of siRNA with Selected Lipopeptides: A Computer-Aided Study of the Effect of Lipopeptides' Functional Groups and Stereoisomerism. J. Chem. Theory Comput. 2020, 16, 3842-3855.

39. Draksharapu, A.; Boersma, A. J.; Leising, M.; Meetsma, A.; Browne, W. R.; Roelfes, G., Binding of copper (II) polypyridyl complexes to DNA and consequences for DNA-based asymmetric catalysis. Dalton Trans. 2015, 44, 3647-3655.

40. Rohs, R.; West, S. M.; Sosinsky, A.; Liu, P.; Mann, R. S.; Honig, B., The role of DNA shape in protein-DNA recognition. Nature 2009, 461, 1248-1253.

41. Gordon, B. R.; Li, Y.; Cote, A.; Weirauch, M. T.; Ding, P.; Hughes, T. R.; Navarre, W. W.; Xia, B.; Liu, J., Structural basis for recognition of AT-rich DNA by unrelated xenogeneic silencing proteins. Proc. Natl. Acad. Sci. USA 2011, 108, 10690-10695.

42. Draksharapu, A.; Boersma, A. J.; Browne, W. R.; Roelfes, G., Characterisation of the interactions between substrate, copper (II) complex and DNA and their role in rate acceleration in DNA-based asymmetric catalysis. Dalton Trans. 2015, 44, 3656-3663.

43. Boersma, A. J.; de Bruin, B.; Feringa, B. L.; Roelfes, G., Ligand denticity controls enantiomeric preference in DNA-based asymmetric catalysis. Chem. Commun. 2012, 48, 2394-2396.

44. Wang, C.; Qi, Q.; Li, W.; Dang, J.; Hao, M.; Lv, S.; Dong, X.; Gu, Y.; Wu, P.; Zhang, W.; Chen, Y.; Hartig, J. S., A Cu(II)-ATP complex efficiently catalyses enantioselective Diels-Alder reactions. Nat. Commun. 2020, 11, 4792.

45. $\quad$ Amirbekyan, K.; Duchemin, N.; Benedetti, E.; Joseph, R.; Colon, A.; Markarian, S. A.; Bethge, L.; Vonhoff, S.; Klussmann, S.; Cossy, J.; Vasseur, J.-J.; Smietana, M., Design, synthesis, and binding affinity evaluation of hoechst 33258 derivatives for the development of sequence-specific DNA-based asymmetric catalysts. ACS Catal. 2016, 6, 3096-3105.

46. Boersma, A. J.; Megens, R. P.; Feringa, B. L.; Roelfes, G., DNA-based asymmetric catalysis. Chemical Society Reviews 2010, 39, 2083-2092.

47. Marek, J. J.; Singh, R. P.; Heuer, A.; Hennecke, U., Enantioselective Catalysis by Using Short, Structurally Defined DNA Hairpins as Scaffold for Hybrid Catalysts. Chem. Eur. J. 2017, 23, 6004-6008.

48. Wang, J.; Benedetti, E.; Bethge, L.; Vonhoff, S.; Klussmann, S.; Vasseur, J. J.; Cossy, J.; Smietana, M.; Arseniyadis, S., DNA vs. mirror-image DNA: a universal approach to tune the absolute configuration in DNA-based asymmetric catalysis. Angew. Chem. Int. Ed. 2013, $125,11760-11763$

49. $\mathrm{Li}, \mathrm{Y} . ;$ Cheng, M.; Hao, J.; Wang, C.; Jia, G.; Li, C., Terpyridine-Cu (II) targeting human telomeric DNA to produce highly stereospecific G-quadruplex DNA metalloenzyme. Chemical Science 2015, 6, 5578-5585.

50. Benedetti, E.; Duchemin, N.; Bethge, L.; Vonhoff, S.; Klussmann, S.; Vasseur, J.-J.; Cossy, J.; Smietana, M.; Arseniyadis, S., DNA-cellulose: An economical, fully recyclable and highly effective chiral biomaterial for asymmetric catalysis. Chem. Commun. 2015, 51, 60766079 .

51. Park, S.; Ikehata, K.; Sugiyama, H., Solid-supported DNA for asymmetric synthesis: a stepping-stone toward practical applications. Biomaterials Science 2013, 1, 1034-1036.

52. Megens, R. P.; Roelfes, G., Organic co-solvents in aqueous DNA-based asymmetric catalysis. Org. Biomol. Chem. 2010, 8, 1387-

1393.

53. Zhao, H.; Shen, K., G-quadruplex DNA-based asymmetric catalysis of michael addition: Effects of sonication, ligands, and cosolvents. Biotechnology Progress 2016, 32, 891-898.

54. Zhao, H.; Shen, K., DNA-based asymmetric catalysis: role of ionic solvents and glymes. RSC advances 2014, 4, 54051-54059.

55. Abe, H.; Abe, N.; Shibata, A.; Ito, K.; Tanaka, Y.; Ito, M.; Saneyoshi, H.; Shuto, S.; Ito, Y., Structure formation and catalytic activity of DNA dissolved in organic solvents. Angew. Chem. 2012, 124, 6581-6585.

56. $\quad$ Percec, V.; Cho, W.-D.; Ungar, G.; Yeardley, D. J., Synthesis and structural analysis of two constitutional isomeric libraries of AB2based monodendrons and supramolecular dendrimers. J. Am. Chem. Soc. 2001, 123, 1302-1315.

57. Kuang, H.; Gartner lii, T. E.; de Mello, M. D.; Guo, J.; Zuo, X.; Tsapatsis, M.; Jayaraman, A.; Kokkoli, E., ssDNA-amphiphile architecture used to control dimensions of DNA nanotubes. Nanoscale 2019, 11, 19850-19861. 